\title{
Regulators of skeletal development: a cluster analysis of 206 bone tumors reveals diagnostically useful markers
}

\author{
Andrew E Horvai ${ }^{1}$, Ritu Roy ${ }^{2}$, Dariusz Borys ${ }^{3}$ and Richard J O’Donnell ${ }^{4}$ \\ ${ }^{1}$ Department of Pathology, University of California, San Francisco, CA, USA; ${ }^{2}$ Department of Biostatistics, \\ UCSF Comprehensive Cancer Center, San Francisco, CA, USA; ${ }^{3}$ Department of Pathology, University of \\ California, Davis, CA, USA and ${ }^{4}$ Department of Orthopaedic Surgery, University of California, San Francisco, \\ CA, USA
}

The molecules Indian hedgehog (IHH), SP7 (also known as osterix), sex-determining region Y-box 9 (SOX9), runt-related transcription factor 2 (RUNX2) and TWIST1 regulate the normal differentiation of osteo- and chondrogenic cells from precursors during skeletal development and remodeling. The aberrant function of the same molecules has been implicated in the pathogenesis of bone tumors. Preliminary studies suggest that antibodies against these molecules have practical, diagnostic or prognostic utility in tumors. However, a comprehensive analysis of the expression of these molecules in a large, diverse set of bone tumors has yet to be reported. The goals of this study were to compare the immunohistochemical profiles of IHH, SP7, SOX9, RUNX2 and TWIST1 among bone tumors and to determine the optimum panel for diagnostic utility. Tissue microarrays prepared from 206 undecalcified tumors (71 osteosarcomas, 26 osteoblastomas/osteoid osteomas, 50 giant cell tumors, 5 chondromyxoid fibromas and 54 chondroblastomas) were stained with antibodies to $\mathrm{IHH}$, SP7, SOX9, RUNX2 and TWIST1. The stains were scored for intensity $(0-3+)$ and distribution. The results were analyzed by cluster analysis. Optimum antibody panels for diagnostic sensitivity and specificity were calculated. Analysis revealed six main clusters that corresponded well to tumor types and suggested a close relationship between the stromal cells of giant cell tumor and the osteoblasts of osteosarcoma. The expression profile of chondromyxoid fibroma and chondroblastoma also suggested related differentiation. The distribution of osteoblastomas and osteoid osteomas was more heterogeneous. RUNX2, SOX9 and TWIST1 represented the most sensitive and specific immunohistochemical panel to distinguish among these diagnoses with the limitation that no result could discriminate between chondroblastoma and chondromyxoid fibroma. IHH and SP7 did not yield additional utility.

Modern Pathology (2012) 25, 1452-1461; doi:10.1038/modpathol.2012.110; published online 6 July 2012

Keywords: bone tumor; immunohistochemistry; tissue microarray

Master regulatory genes control the differentiation of multipotent stem cells into divergent mature mesenchymal cells. The lineage-specific expression of master regulators has allowed their use for diagnostic purposes in mesenchymal tumors. For example, immunohistochemical detection of the transcription factors myogenin and peroxisome proliferator gamma are valuable adjuncts to the diagnosis of

Correspondence: Dr A Horvai, MD, PhD, Department of Pathology, University of California, 1600 Divisadero Drive B220, San Francisco, CA 94115-1656, USA.

E-mail: andrew.horvai@ucsf.edu

Received 7 February 2012; revised 2 May 2012; accepted 14 May 2012; published online 6 July 2012 rhabdomyosarcomas and liposarcomas, respectively. ${ }^{1,2}$ Such tests are particularly helpful to interpret small biopsies from minimally invasive procedures in soft tissue sarcomas.,

In contrast to soft tissue tumors, the majority of bone tumors lack widely used immunohistochemical stains. The rarity and intratumoral heterogeneity of bone tumors and the sensitivity of some antigens to decalcification impede widespread use of immunohistochemistry. Although the diagnosis of some bone tumors can be made from routine hematoxylin and eosin-stained slides with radiographic correlation, the significant morphologic overlap among tumors mandate larger, more invasive intralesional biopsies. Specifically, osteosarco- 
Table 1 Antibodies used

\begin{tabular}{|c|c|c|c|c|c|}
\hline Antibody & Source & City, state & Pre-treatment & Dilution & Incubation \\
\hline $\mathrm{IHH}$ & Santa Cruz & Santa Cruz, CA & DAKO pressure cooker, & $1: 100$ & $16 \mathrm{~h} 4{ }^{\circ} \mathrm{C}$ \\
\hline SP7 & Santa Cruz & Santa Cruz, CA & citrate $\mathrm{pH} 6,40 \mathrm{~min}$ & $1: 50$ & \\
\hline SOX9 & Abcam & Cambridge, MA & & $1: 50$ & \\
\hline RUNX2 & Sigma & St Louis, MO & & $1: 100$ & \\
\hline TWIST1 & Abcam & Cambridge, MA & & $1: 400$ & \\
\hline
\end{tabular}

mas may contain abundant osteoclasts, ${ }^{5}$ chondroid or fibrochondroid matrix ${ }^{6,7}$ or primitive small cells, ${ }^{8}$ thus mimicking giant cell tumor, chondromyxoid fibroma, chondroblastoma or Ewing sarcoma, respectively. The immunohistochemical detection of bone matrix proteins has some utility in the diagnosis of osteosarcoma, but the specificity to exclude other matrix-producing tumors is less clear. $^{9-12}$

Recently, improved understanding of the intracellular mechanisms of skeleton formation and remodeling has revealed lineage-specific signaling molecules. The mechanisms are complex and include combinatorial interactions of multiple molecules promoting osteoblast and chondrocyte differentiation. ${ }^{13-17}$ Nevertheless, studies suggest that the signal transduction molecule Indian hedgehog (IHH), and the transcription factors SP7 (also known as osterix, OSX), sex-determining region Y-box 9 (SOX9), runt-related transcription factor 2 (RUNX2, also known as CBFA1) and TWIST1 regulate the differentiation of osteogenic and chondrogenic cells from mesenchymal precursors. ${ }^{13,18-21}$ Furthermore, the aberrant function of these same molecules has also been implicated in the pathogenesis of some bone tumors including osteosarcoma and giant cell tumor. $^{22-24}$ Preliminary studies suggest that antibodies against these molecules have diagnostic or prognostic utility in benign and malignant bone tumors. $^{25-28}$ However, a comprehensive analysis using a large number of diverse bone tumors has yet to be studied for the utility of immunohistochemistry of these markers.

In order to better characterize the expression and diagnostic utility of IHH, SP7, SOX9, RUNX2 and TWIST1 in bone tumors, we evaluated 206 tumors using tissue microarrays by immunohistochemistry. We report that hierarchical cluster analysis of the expression data sorts tumors into established diagnostic categories, with some interesting exceptions. Moreover, the optimal diagnostic panel of SOX9, RUNX2 and TWIST1 can aid in discriminating between these tumors.

\section{Materials and methods}

\section{Case Selection and Array Construction}

A total of 206 tumors were identified from the pathology database (71 osteosarcomas, 26 osteoblas- tomas/osteoid osteomas, 50 giant cell tumors, 5 chondromyxoid fibromas and 54 chondroblastomas) based on available tumor volume. All diagnoses were based on current criteria after review of available histology and radiology. ${ }^{29}$ Osteosarcomas were further subclassified as chondroblastic, osteoblastic, fibroblastic, small cell, and low grade (central, parosteal and periosteal). A subset of the giant cell tumors and osteosarcomas have been previously reported by us. ${ }^{30,31}$ Cores of formalin fixed, paraffin-embedded tissue were incorporated in duplicate or triplicate into recipient paraffin blocks as previously described. ${ }^{32}$ Care was taken to avoid cores of heavily mineralized matrix, by targeting more cellular areas. A relatively large core size $(3 \mathrm{~mm})$ was chosen for this array, with the tradeoff of a larger number of recipient blocks for the following reasons: the heterogeneity of cellularity and matrix composition in many of the tumors could result in thin (ie, $1 \mathrm{~mm}$ ) cores sampling areas rich in matrix with too few cells for study; microtome sectioning of undecalcified bone incorporated into arrays is technically challenging but was more consistent with larger cores; larger cores more closely approximate the total tissue cross-sectional area from needle core biopsies.

\section{Immunohistochemistry}

Four $\mu \mathrm{m}$ sections were stained with antibodies (Table 1) using standard techniques. Detection for all cases was performed by Vector Labs (Burlingame, CA, USA) ABC kit according to the manufacturer's directions. The ABC detection method was chosen to be congruent with the methods of prior publications using these antibodies and for economic reasons. The intensity, pattern and distribution of staining were recorded. Staining was evaluated according to the four-point system of Adams et al $\mathrm{l}^{33}$ as follows:

- Strong $(3+)$ : dark staining that is easily visible at low power and involves $>50 \%$ of cells.

- Moderate $(2+)$ : focal darkly staining areas $(<50 \%$ of cells) or moderate staining of $>50 \%$ of cells.

- Weak $(1+)$ : focal moderate staining in $<50 \%$ of cells, or pale staining in any proportion of cells not easily seen at low power.

- Negative $(0+)$ : none of the above. 
Positive staining was defined as cytoplasmic and/ or nuclear for $\mathrm{IHH}$, and nuclear for the remaining antigens although the presence of cytoplasmic staining was noted if moderate or strong. The distribution of staining (spindle cells, osteoblasts and osteoclasts) was recorded, particularly in the more heterogeneous tumors (chondroblastic osteosarcoma, giant cell tumor and chondroblastoma). Normal growth plate was used as positive control.

\section{Statistics}

Detailed statistical methods are described in Supplementary Materials. Briefly, we performed unsupervised clustering of samples with Euclidean distance and Ward's linkage method. We applied three different approaches to identify antibodies to predict tumor type: (1) regularized logistic regression, with lasso regularization and 10 -fold cross validation, ${ }^{34}(2)$ deviance loss measure and (3) 'onestandard-error' rule. All analysis was performed using the statistical software known as ' $R$ ' ( $R$ development team, Vienna, Austria). ${ }^{35}$ Only those antibodies that were identified by all three methods were analyzed further with the exception of chondromyxoid fibroma. In these tumors, RUNX2 met the above requirements whereas SOX9 showed significant correlation with the diagnosis in univariate logistic regression. We acknowledge that there were very few cases of some tumor types, especially chondromyxoid fibroma, to be able to develop robust predictors.

\section{Results}

\section{Immunohistochemistry}

Representative immunohistochemical results are illustrated in Figures 1 and 2 and summarized in Supplementary Table 2. Staining scores between duplicate cores showed $>98 \%$ concordance (four discordant cases each for IHH and SP7, respectively, and one discordant case for TWIST1). Consistent nuclear expression was observed for SOX9 in chondromyxoid fibromas $(5 / 5,100 \%)$ and chondroblastomas $(53 / 54,98 \%)$. In all cases, the expression was restricted to the mononuclear round or stellate cells and absent in osteoclast-type giant cells. All chondroblastic osteosarcomas $(23 / 23,100 \%)$ were also positive for SOX9, in the chondrogenic component, although a smaller proportion of the remaining osteosarcomas $(18 / 54,33 \%)$ showed positive staining in malignant osteoblasts. Small cell osteosarcomas $(n=3)$, however, were uniformly negative. Moderate-to-strong $(2-3+) \mathrm{IHH}$ expression was noted only in osteosarcomas $(17 / 71,24 \%)$, rare chondroblastomas $(2 / 54,4 \%)$ and in a subset of the mononuclear cells $(<10 \%$ of total cell volume) of giant cell tumors (Figure 1). Some weak staining was also noted in the osteoclast-type giant cells in a variety of tumors.
SP7 staining was, at most, moderate $(2+)$ in the few positive cases and restricted predominantly to osteosarcomas $(6 / 71,8 \%)$. Chondromyxoid fibromas were uniformly negative for this marker and only a single chondroblastoma was positive. Moderateto-strong $(2-3+)$ RUNX2 expression was most common in osteoblastomas (19/21, 90\%) osteoid osteomas $(5 / 5,100 \%)$ and osteosarcomas $(66 / 71,93 \%)$. Intriguingly, a subset of the mononuclear cells of giant cell tumors consistently stained strongly for RUNX2 (49/50, 98\%) but staining was absent in the osteoclasts of giant cell tumors (Figure 1). Strongest TWIST1 expression $(\geq 2+)$ was in osteosarcomas $(60 / 71,85 \%)$ and osteoblastomas/osteoid osteomas $(12 / 26,50 \%)$.

\section{Cluster Analysis}

The relationship between expression patterns using unsupervised hierarchical cluster analysis revealed six main clusters (Figure 3) that corresponded well to tumor types, with some exceptions. Clusters A and $B$ represented the majority (46/50, 92\%) of giant cell tumors. However, cluster A also contained a subset of osteosarcomas, predominantly chondroblastic $(n=5)$ and low grade (parosteal and central, $n=3$ ), subtypes. Clusters $C$ and D consisted almost entirely of high-grade osteosarcomas. Chondromyxoid fibromas and chondroblastomas formed cluster E. Cluster $\mathrm{F}$ was a mixture of tumors that were, at most, weakly positive for most markers.

\section{Sensitivity and Specificity of Immunostaining Results}

The optimum diagnostic panel of antibodies and their sensitivity and specificity in the diagnosis of these tumors are summarized in Table 2. The analysis (see Materials and methods section) resulted in highest sensitivity and specificity when the threshold of staining score $\geq 2$ was set as a positive result and $\leqslant 1$ as negative. RUNX2, TWIST1 and SOX9 were the most useful antibodies in distinguishing among these diagnoses whereas $\mathrm{IHH}$ and SP7 showed less utility.

Giant cell tumor can be separated from the remaining diagnoses with positive RUNX2 and negative TWIST1 (sensitivity 90\% and specificity $83 \%$ ). The combination of RUNX2 and TWIST1 positivity points to osteosarcoma (sensitivity $82 \%$ and specificity $86 \%$ ), regardless of subtype. In contrast, the combination of a RUNX2-negative and SOX9-positive result points to either chondroblastoma (sensitivity $53 \%$ and specificity $99 \%$ ) or chondromyxoid fibroma (sensitivity $80 \%$ and specificity $85 \%$ ). Although the specificity is still relatively good when considering all the diagnoses studied, it should be noted that the (SOX9+ and RUNX2-) immunophenotype is shared between chondromyxoid fibroma and chondroblastoma so this panel is not useful if the differential diagnosis 
$\mathrm{IHH}$
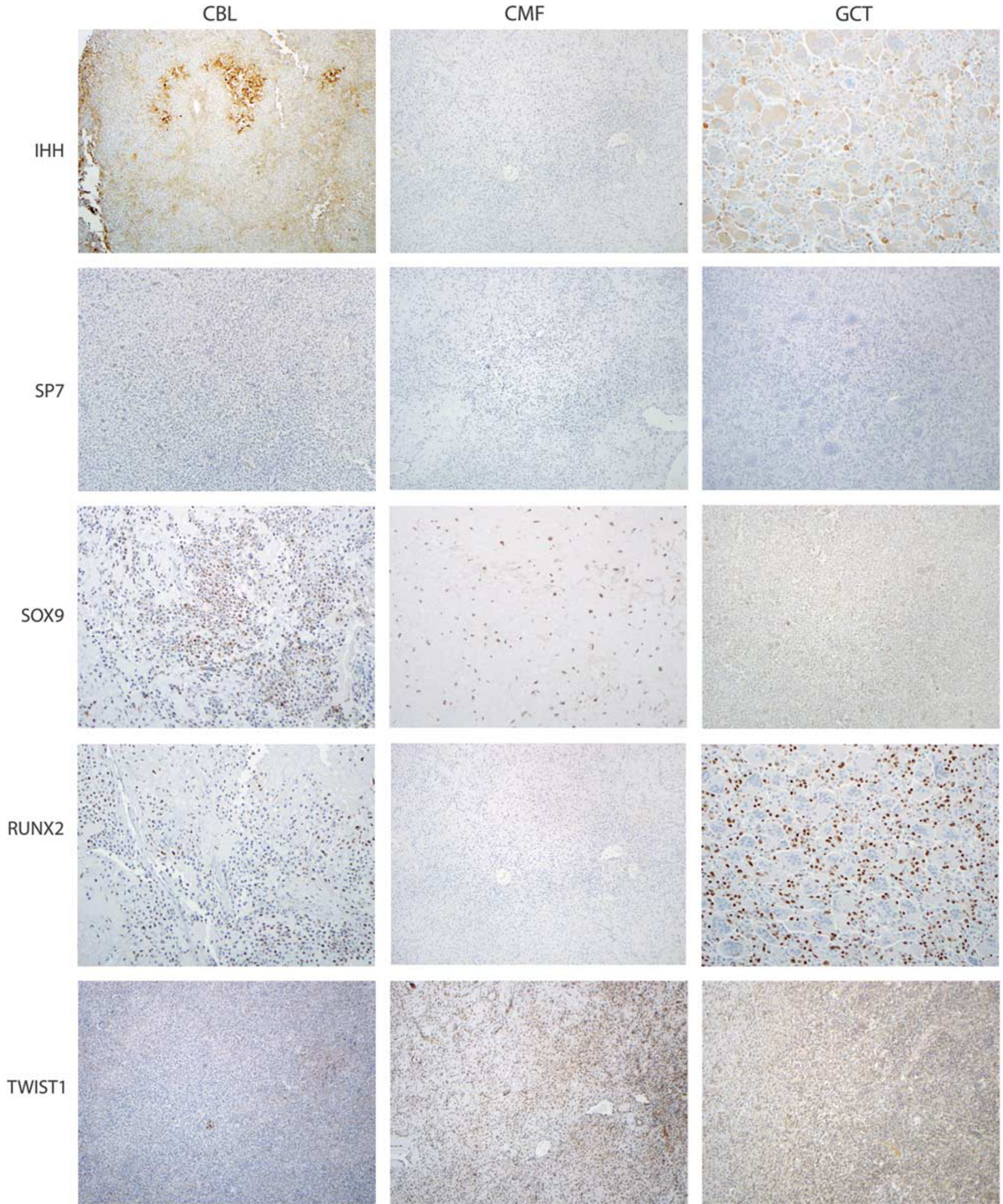

Figure 1 Representative immunohistochemical staining of chondroblastic and giant cell tumors. IHH showed nuclear and cytoplasmic staining, albeit weak, predominantly in giant cell tumors. Nuclear SP7 was expressed in very few tumors, and only a single chondroblastoma. Nuclear SOX9 was most common in the chondromyxoid fibromas, chondroblastomas. RUNX2 was present in the stromal cells of giant cell tumors. CBL, chondroblastoma; CMF, chondromyxoid fibroma; GCT, giant cell tumor; IHH, Indian hedgehog.

only includes the above two entities. Finally, as suggested by the heterogeneous immunophenotype of osteoblastomas and osteoid osteomas on the heatmap (Figure 3), a diagnostic antibody panel with good specificity and sensitivity could not be identified. 

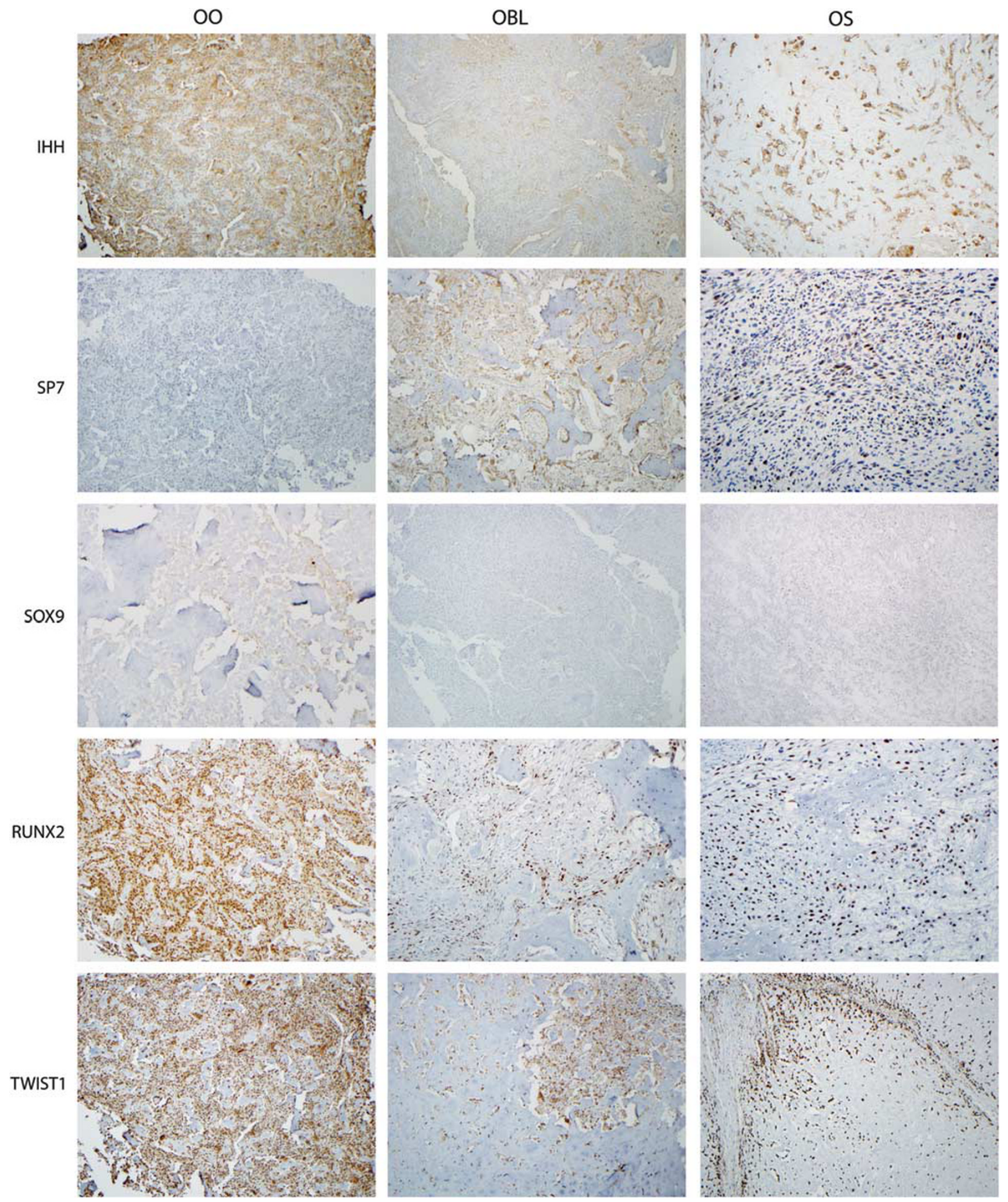

Figure 2 Representative immunohistochemical staining of osteoblastic tumors. IHH showed nuclear and cytoplasmic staining in most osteoblastic neoplasms. Nuclear SP7 was largely absent in this group of tumors. Nuclear SOX9 was typically absent in osteoblastic tumors except rare chondroblastic osteosarcomas. Osteosarcomas expressed RUNX2 and frequently co-expressed TWIST1. IHH, Indian hedgehog; OBL, osteoblastoma; OO, osteoid osteoma; OS, osteosarcoma.

\section{Discussion}

The diagnosis of bone tumors requires careful correlation between histologic, radiographic and clinical parameters. The combination of significant morphologic overlap between bone tumors, intratumoral heterogeneity and the preference for smaller, less invasive, biopsies increases the challenge faced 

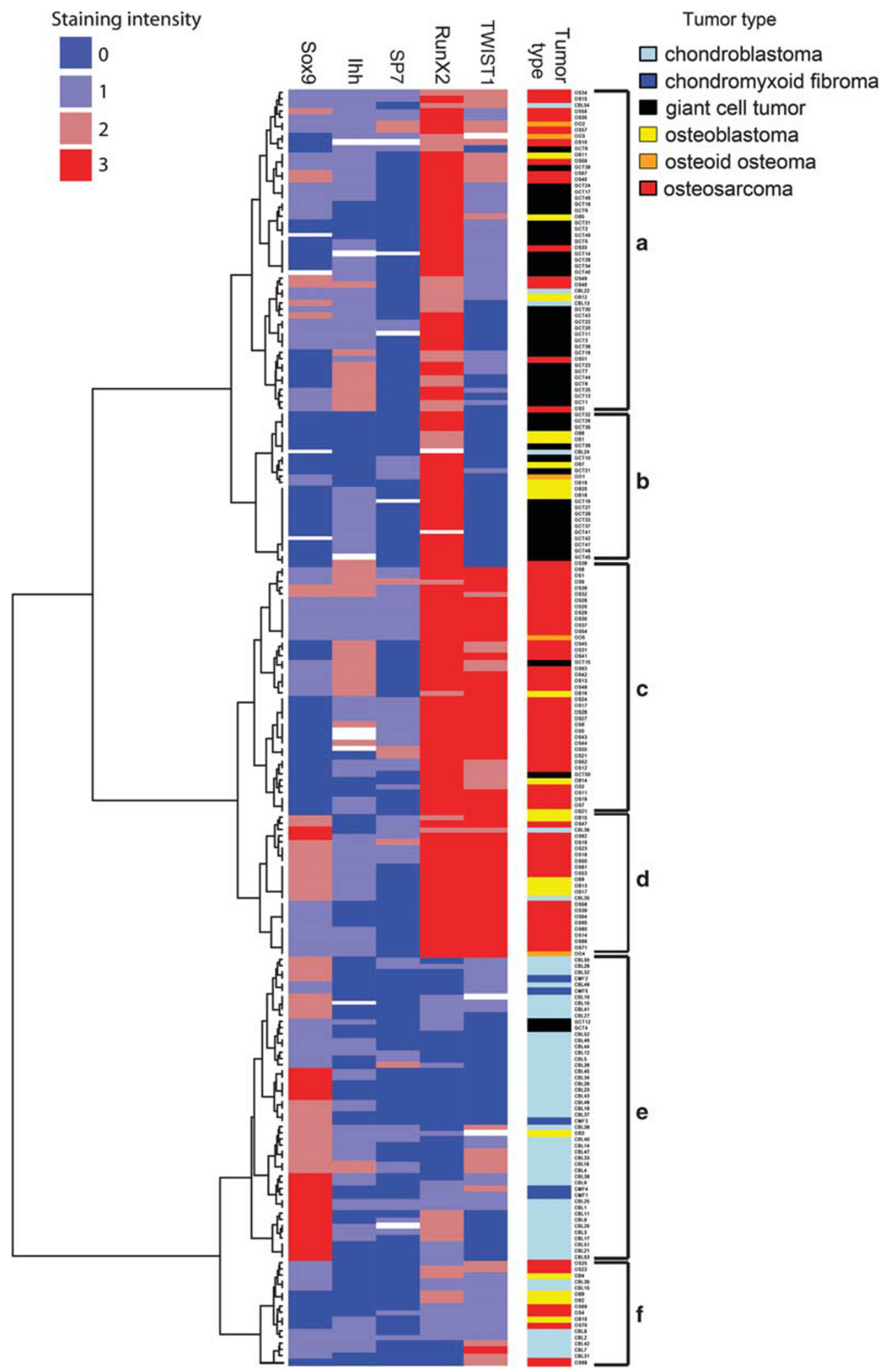

Tumor type

taining intensity

a

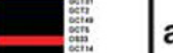

b

C

d

e

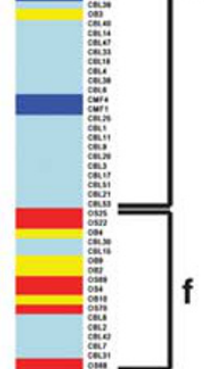

Figure 3 Heat map of immunohistochemical data. Major clusters (a-f), based on the third division, are demonstrated to the right and antigens tested along the top. Staining score is indicated by color (see legend, a white bar indicates data not available). The right column gives tumor type by color (see legend) and also by specific tumor number. CMF, chondromyxoid fibroma; CBL, chondroblastoma; GCT, giant cell tumor; IHH, Indian hedgehog; OS, osteosarcoma; OO, osteoid osteoma; OB, osteoblastoma; RUNX2, runt-related transcription factor 2; SOX9, sex-determining region Y-box 9. 
Table 2 Sensitivity and specificity of antibody panels for each diagnosis

\begin{tabular}{llcr}
\hline Diagnosis & Optimum panel & Sensitivity (\%) & Specificity (\%) \\
\hline Giant cell tumor & RUNX2+, TWIST1 - & 90 & 83 \\
Osteosarcoma & RUNX2+, TWIST1+ & 82 & 86 \\
Chondroblastoma & RUNX2-, SOX9+ & 53 & 99 \\
Chondromyxoid fibroma & RUNX2-, SOX9+ & 80 & 85 \\
\hline
\end{tabular}

by pathologists. This study demonstrates that immunohistochemical expression profiling and unsupervised cluster analysis categorizes bone tumors into existing histologic categories and highlights optimum diagnostic antibody panels. The expression of SOX9, SP7, RUNX2, TWIST1 and IHH was tested because these molecules serve as regulators of normal skeletal development and control lineagespecific tumor differentiation. ${ }^{13-17}$ Unlike cDNA expression profiling, which reports the pooled molecular signature of all cells within a tumor, immunohistochemical profiling reveals the intratumoral distribution of protein expression, an advantage in polymorphic cellular populations.

The separation of osteoblastic (clusters A-C) and chondroblastic (cluster D) tumors represents the most fundamental division extracted from the data. Although this classification is perhaps not surprising for tumors in which osteoblastic differentiation is definitional (ie, osteosarcoma), it does clarify the lineage in tumors where this is less clear. For example, chondroblastoma and chondromyxoid fibroma rarely produce normal hyaline cartilage, whereas some biochemical studies have even suggested that the matrix proteins in the former are comparable to osteoid thus raising questions about the nature of the tumor cells. ${ }^{36}$ However, based on the expression of SOX9, an early regulator of chondrogenesis $^{37}$ and absence of RUNX2, an early marker of osteoblastic differentiation ${ }^{38-40}$ our findings support the correct classification of chondroblastoma and chondromyxoid fibroma along the chondroblastic lineage. That the above two tumor types could not be separated using the available markers, despite distinct histopathology in most cases, suggests convergent differentiation pathways.

The link of giant cell tumor to the osteoblastic clusters supports the current view that the stromal cells in these tumors are, in fact, primitive osteoblast precursors, despite the absence of osteoid synthesis. ${ }^{41,42}$ The pattern of staining with RUNX2 in only a few stromal cells of GCT supports that the neoplastic cells are likely only small component of a heterogeneous population. ${ }^{23,41}$ Interestingly, predominantly low-grade osteosarcomas clustered together with giant cell tumors. This observation implies that neoplastic cells of low-grade osteosarcomas and stromal cells of giant cell tumor may be closely related, possibly at an earlier phase of osteoblastic differentiation.

Osteoid osteomas and osteoblastomas, although largely restricted to the osteoblastic clusters (clusters A-C), were heterogeneously distributed between these clusters rather than forming a single, well-defined, group. The majority of osteoblastomas were positive for RUNX2, in keeping with prior observations ${ }^{25}$ and their presumed osteoblastic lineage, but the expression of the remaining markers was less predictable than for other tumor types. For osteoid osteoma, at least, some of the variability can be attributed to the limited number of cases available. In osteoblastomas, which were more numerous, however, the results suggest true heterogeneity of the molecular signature. That is, some osteoblastomas resemble the stromal cells of giant cell tumors and low-grade osteosarcomas, whereas other osteoblastomas resemble high-grade osteosarcomas. Whether a difference in clinical outcome exists between osteoblastomas that cluster with high-grade osteosarcomas and those that cluster with giant cell tumors and low-grade osteosarcomas remains to be determined.

The second major aim of this study was to identify specific diagnostic antibody panels that may be useful in difficult cases, especially on small biopsies. Osteosarcoma, giant cell tumor, chondromyxoid fibroma and chondroblastoma may be relatively easy to diagnose when classic morphology and radiographic findings are present. However, a prominent giant cell component, chondroid or chondromyxoid stroma can blur the distinction between these diagnoses. ${ }^{5,6}$ Although the presence of osteoid production by tumor cells is a defining feature of osteosarcoma, it may be difficult to identify on small biopsies. No single diagnostic marker, used alone, was specific for any tumor type, supporting that combinatorial interactions between multiple transcription factors drive the phenotypes of currently accepted tumor categories. The initial statistical analysis from the hierarchical data yielded best diagnostic sensitivity and specificity when only moderate or strong, diffuse $(\geq 2+)$ staining was categorized 'positive'. A high threshold for a positive result confers the added benefit of more straightforward interpretation in routine practice.

The combination of RUNX2 and TWIST1 immunopositivity is the most sensitive ( $82 \%$ ) and specific $(86 \%)$ panel for osteosarcoma whereas RUNX2 without TWIST1 positivity supports giant cell tumor $(90 \%$ sensitivity and $83 \%$ specificity). The functional significance of RUNX2 has also been proposed in the stromal cells of giant cell tumor ${ }^{23}$ 
paralleling our observation of RUNX2 staining in a subset of the mononuclear cells. TWIST1 is a transcription factor that has also been shown to regulate early osteoblastic differentiation and downregulates RUNX2 in giant cell tumor. ${ }^{13,14,24}$ Although TWIST1 appears to be expressed in cultured stromal cells of giant cell tumor at the RNA level, our results indicate that the level of protein expression may be lower than that observed in osteosarcoma. ${ }^{24}$ Alternatively, the expression pattern of TWIST1 in cultured cells may not mimic those in intact tissue.

SOX9 expression, in the absence of RUNX2, proved the most useful immunophenotype to support chondromyxoid fibroma (sensitivity $80 \%$ and specificity $85 \%$ ) and chondroblastoma (sensitivity 53\% and specificity 99\%) over the remaining diagnoses. RUNX2, as mentioned above, regulates differentiation along the osteoblastic pathway but may also promote chondrocyte maturation in normal tissues. ${ }^{20,43}$ In past studies, RUNX2 expression was observed in hyaline cartilage tumors but not in chondroblastoma and chondromyxoid fibroma, matching our own observations. $^{25,27}$ Thus, the latter two tumors may recapitulate an earlier stage of maturation along the chondrocyte lineage when RUNX2 is not yet highly expressed. We did observe that many osteosarcomas, especially, but not exclusively, chondroblastic subtypes, co-expressed RUNX2 and SOX9. SOX9 may be suppressed in the osteoblastic lineage during normal differentiation, but in osteosarcomas, especially chondroblastic ones, may contain bipotential cells capable of both chondroblastic and osteoblastic differentiation. $^{20,44}$ Importantly, no combination of antibodies distinguished between chondroblastoma and chondromyxoid fibroma suggesting a close molecular relationship between these tumors. Furthermore, no specific panel could confirm the diagnoses of osteoblastoma and osteoid osteoma. Several reasons may explain this limitation of the data. First, as discussed above, osteoblastomas were more heterogeneously distributed in the cluster analysis. Second, osteoblastomas were disproportionately represented in the cluster of tumors negative for most or all markers, perhaps indicating poor antigen preservation. We specifically avoided incorporating decalcified tissue into the arrays, but cannot exclude that other tissue processing effects affected antigenicity in this group.

IHH is normally expressed in hypertrophic chondrocytes of the growth plate and is thought to lie downstream of RUNX2 in the signaling cascade. IHH expression has been reported in osteosarcomas, chondroblastomas and hyaline cartilage-producing tumors. ${ }^{27,28,45-47}$ Its expression in osteoblastoma, chondromyxoid fibroma and giant cell tumor, as demonstrated in our study, has not been previously reported. The widespread, albeit weak, expression of IHH suggests a functional role in multiple lineages. Indeed, the primitive cells of Ewing sarcoma are the only primary bone tumors that we have observed, thus far, to be consistently negative for $\mathrm{IHH}(\mathrm{AH}-$ unpublished observations). The relatively ubiquitous, weak expression, without strong expression in any tumor type in the present series did not provide added diagnostic utility.

SP7, has been detected in giant cell tumor at the RNA level, and osteoblastic but not chondroblastic neoplasms. ${ }^{22,25}$ Functionally, SP7 may suppress SOX9 expression and thereby direct cells to an osteoblastic fate. In our series, SP7 was expressed in nearly all osteoblastic tumors but was largely absent in giant cell tumor, chondroblastoma and chondromyxoid fibroma. However, even in positive cases, staining was usually weak and thus did not improve the sensitivity or specificity of diagnostic panels. Interestingly, the single chondroblastoma (CBL26), which did show moderate $(2+)$ SP7 expression displayed more aggressive clinical behavior (multiple, destructive recurrences) raising the possibility that this case might represent a higher grade form of the tumor. ${ }^{6}$

In summary, in this study we demonstrate that the expression patterns of skeletal development signaling molecules reinforce current diagnostic categories of bone tumors. Cluster analysis suggests a molecular relationship between a subset of osteosarcomas and the stromal cells of giant cell tumors. RUNX2, SOX9 and TWIST1 represent the most useful immunohistochemical panel to distinguish among these diagnoses with reasonable specificity and sensitivity although the results need to be interpreted in the correct histologic and radiographic context.

\section{Acknowledgement}

We thank Loretta Chan and the Immunopathology core at the UCSF Comprehensive Cancer Center and the staff of the UCSF Histology Laboratory for their technical assistance.

\section{Disclosure/conflict of interest}

The authors declare no conflict of interest.

\section{References}

1 Folpe AL. MyoD1 and myogenin expression in human neoplasia: a review and update. Adv Anat Pathol 2002;9:198-203.

2 Horvai AE, Schaefer JT, Nakakura EK, et al. Immunostaining for peroxisome proliferator gamma distinguishes dedifferentiated liposarcoma from other retroperitoneal sarcomas. Mod Pathol 2008;21:517-524.

3 Ordonez NG. Application of immunocytochemistry in the diagnosis of soft tissue sarcomas: a review and update. Adv Anat Pathol 1998;5:67-85.

4 Coindre JM. Immunohistochemistry in the diagnosis of soft tissue tumours. Histopathology 2003;43:1-16. 
5 Bathurst N, Sanerkin N, Watt I. Osteoclast-rich osteosarcoma. Br J Radiol 1986;59:667-673.

6 Bacchini P, Inwards C, Biscaglia R, et al. Chondroblastoma-like osteosarcoma. Orthopedics 1999;22: 337-339.

7 Schajowicz F, de Prospero JD, Cosentino E. Case report 641: chondroblastoma-like osteosarcoma. Skeletal Radiol 1990;19:603-606.

8 Nakajima H, Sim FH, Bond JR, et al. Small cell osteosarcoma of bone. Review of 72 cases. Cancer 1997; 79:2095-2106.

9 Wagner ER, Luther G, Zhu G, et al. Defective osteogenic differentiation in the development of osteosarcoma. Sarcoma 2011;2011:325238.

10 Mayordomo E, Machado I, Giner F, et al. A tissue microarray study of osteosarcoma: histopathologic and immunohistochemical validation of xenotransplanted tumors as preclinical models. Appl Immunohistochem Mol Morphol 2010;18:453-461.

11 Fanburg JC, Rosenberg AE, Weaver DL, et al. Osteocalcin and osteonectin immunoreactivity in the diagnosis of osteosarcoma. Am J Clin Pathol 1997;108: 464-473.

12 Chano T, Matsumoto K, Ishizawa M, et al. Analysis of the presence of osteocalcin, S-100 protein, and proliferating cell nuclear antigen in cells of various types of osteosarcomas. Eur J Histochem 1996;40: 189-198.

13 Lee MS, Lowe GN, Strong DD, et al. TWIST, a basic helix-loop-helix transcription factor, can regulate the human osteogenic lineage. J Cell Biochem 1999;75: 566-577.

14 Jabs EW. A TWIST in the fate of human osteoblasts identifies signaling molecules involved in skull development. J Clin Invest 2001;107:1075-1077.

15 Mackie EJ, Ahmed YA, Tatarczuch L, et al. Endochondral ossification: how cartilage is converted into bone in the developing skeleton. Int J Biochem Cell Biol 2008;40:46-62.

16 Zhang C. Transcriptional regulation of bone formation by the osteoblast-specific transcription factor Osx. J Orthop Surg Res 2010;5:37.

$17 \mathrm{He} \mathrm{N}$, Xiao Z, Yin T, et al. Inducible expression of RunX2 results in multiorgan abnormalities in mice. J Cell Biochem 2011;112:653-665.

18 St-Jacques B, Hammerschmidt M, McMahon AP. Indian hedgehog signaling regulates proliferation and differentiation of chondrocytes and is essential for bone formation. Genes Dev 1999;13:2072-2086.

19 Nakashima K, Zhou X, Kunkel G, et al. The novel zinc finger-containing transcription factor osterix is required for osteoblast differentiation and bone formation. Cell 2002;108:17-29.

20 Akiyama H, Kim JE, Nakashima K, et al. Osteochondroprogenitor cells are derived from Sox9 expressing precursors. Proc Natl Acad Sci USA 2005;102:14665-14670.

21 Komori T. RunX2, a multifunctional transcription factor in skeletal development. J Cell Biochem 2002;87:1-8.

22 Huang L, Teng XY, Cheng YY, et al. Expression of preosteoblast markers and Cbfa-1 and Osterix gene transcripts in stromal tumour cells of giant cell tumour of bone. Bone 2004;34:393-401.

23 Mak IW, Cowan RW, Popovic S, et al. Upregulation of MMP-13 via RunX2 in the stromal cell of Giant Cell Tumor of bone. Bone 2009;45:377-386.
24 Singh S, Mak IWY, Cowan RW, et al. The role of TWIST as a regulator in giant cell tumor of bone. J Cell Biochem 2011;112:2287-2295.

25 Dancer JY, Henry SP, Bondaruk J, et al. Expression of master regulatory genes controlling skeletal development in benign cartilage and bone forming tumors. Hum Pathol 2010;41:1788-1793.

26 Andela VB, Siddiqui F, Groman A, et al. An immunohistochemical analysis to evaluate an inverse correlation between RunX2/Cbfa1 and NF kappa B in human osteosarcoma. J Clin Pathol 2005;58:328-330.

27 Park HR, Park YK. Differential expression of RunX2 and Indian hedgehog in cartilaginous tumors. Pathol Oncol Res 2007;13:32-37.

28 Won KY, Park HR, Park YK. Prognostic implication of immunohistochemical RunX2 expression in osteosarcoma. Tumori 2009;95:311-316.

29 Fletcher CDM, Unni KK, Mertens F, (eds). Pathology and Genetics of Tumours of Soft Tissue and Bone. IARC Press: Lyon, 2002.

30 Horvai AE, Kramer MJ, Garcia JJ, et al. Distribution and prognostic significance of human telomerase reverse transcriptase (hTERT) expression in giant-cell tumor of bone. Mod Pathol 2008;21:423-430.

31 Borys D, Canter RJ, Hoch B, et al. P16 expression predicts necrotic response among osteosarcoma patients receiving neoadjuvant chemotherapy. Human Pathol; advance online publication, 9 May 2012; e-pub ahead of print; PMID:22518565.

32 Horvai AE, Kramer MJ, O’Donnell R. Beta-catenin nuclear expression correlates with cyclin D1 expression in primary and metastatic synovial sarcoma: a tissue microarray study. Arch Pathol Lab Med 2006;130:792-798.

33 Adams EJ, Green JA, Clark AH, et al. Comparison of different scoring systems for immunohistochemical staining. J Clin Pathol 1999;52:75-77.

34 Friedman J, Hastie T, Tibshirani R. Regularization paths for generalized linear models via coordinate descent. J Stat Softw 2010;33:1-22.

35 RDevelopmentCoreTeam. R: A Language and Environment for Statistical Computing Vol., R Foundation for Statistical Computing: Vienna, Austria, 2005.

36 Aigner T, Loos S, Inwards C, et al. Chondroblastoma is an osteoid-forming, but not cartilage-forming neoplasm. J Pathol 1999;189:463-469.

37 Wehrli BM, Huang W, De Crombrugghe B, et al. Sox9, a master regulator of chondrogenesis, distinguishes mesenchymal chondrosarcoma from other small blue round cell tumors. Hum Pathol 2003;34:263-269.

38 Ducy P, Zhang R, Geoffroy V, et al. Osf2/Cbfa1: a transcriptional activator of osteoblast differentiation. Cell 1997;89:747-754.

39 Komori T, Yagi H, Nomura S, et al. Targeted disruption of Cbfa1 results in a complete lack of bone formation owing to maturational arrest of osteoblasts. Cell 1997; 89:755-764.

40 Otto F, Thornell AP, Crompton T, et al. Cbfa1, a candidate gene for cleidocranial dysplasia syndrome, is essential for osteoblast differentiation and bone development. Cell 1997;89:765-771.

41 Goldring SR, Roelke MS, Petrison KK, et al. Human giant cell tumors of bone identification and characterization of cell types. J Clin Invest 1987;79: 483-491.

42 Salerno M, Avnet S, Alberghini M, et al. Histogenetic characterization of giant cell tumor of bone. Clin Orthop Relat Res 2008;466:2081-2091. 
43 Takeda S, Bonnamy JP, Owen MJ, et al. Continuous expression of Cbfa1 in nonhypertrophic chondrocytes uncovers its ability to induce hypertrophic chondrocyte differentiation and partially rescues Cbfa1-deficient mice. Genes Dev 2001;15:467-481.

44 Nakashima K, de Crombrugghe B. Transcriptional mechanisms in osteoblast differentiation and bone formation. Trends Genet 2003;19:458-466.

45 Romeo S, Oosting J, Rozeman LB, et al. The role of noncartilage-specific molecules in differentiation of cartilaginous tumors: lessons from chondroblastoma and chondromyxoid fibroma. Cancer 2007;110:385-394.

46 Romeo S, Bovee JV, Jadnanansing NA, et al. Expression of cartilage growth plate signalling molecules in chondroblastoma. J Pathol 2004;202:113-120.

47 Kindblom JM, Nilsson O, Hurme T, et al. Expression and localization of Indian hedgehog (IHH) and parathyroid hormone related protein (PTHrP) in the human growth plate during pubertal development. J Endocrinol 2002;174:R1-R6.

Supplementary Information accompanies the paper on Modern Pathology website (http://www.nature.com/ modpathol) 